\title{
Effect of Foliar Application with Faba Bean and Chickpeas Seed Sprout Extract on Growth and Fruiting of Ewaise Mango Trees
}

"El-Salhy, A.M. ' H.H. Saeed"; A.Y. Ahmed ${ }^{3}$ and I.A. Hassan ${ }^{3}$ CrossMark

${ }^{1}$ Pomology Dept., Fac. Agric., Assiut Univ., Assiut, Egypt.

${ }^{2}$ Hort. Dept., Fac. of Agric. \& Natural Resources, Aswan Univ., Aswan, Egypt.

${ }^{3}$ Agricultural Research Centre, Horticultural Research Institute-Giza.

*E-Mail: abdelfattah.elsalthy@agr.aun.edu.eg

Accepted for publication on: 5/5/2021

\section{Abstract}

An experiment was conducted during two successive seasons 2019 and 2020 on mango trees cv. Ewaise to study the effect of foliar application faba bean and chickpea seed sprout extract at 0.25 to $1 \%$ applied three times along each season (at growth start, fruit set after immediately and one month later) either each extract was sprayed. The influence was evaluated through the response of vegetative growth, leaf $\mathrm{N}, \mathrm{P}, \mathrm{K}$ content, yield and some fruit physical and chemical characteristics.

The obtained results showed that, spaying faba bean and chickpea seed sprout extract at 0.25 to $1 \%$ significantly improved the growth traits and nutritional status of trees compared to untreated one. Also, spray treatments significantly increased yield and improved the fruit quality. No significant difference seen in these studied traits due to increase concentration of used extract from 0.5 to $1 \%$. Therefore from economical point view it could be to use faba bean or chickpea seed sprout at $0.5 \%$. Using chickpea seed sprout extracts spray had a noticeable positive effect particularly on some fruit physical and chemical characteristics than using faba bean seed sprout extracts. Therefore, use two plant seed sprout extracts could be safely recommended as a natural biostimulants application for improving growth and fruiting of Ewaise mango trees grown under the same experimental condition.

\section{Keywords: Seed sprout, mango, yield, fruit quality, environmental pollution.}

\section{Introduction}

Mango (Mangifera indica L.) belongs to the family Anacardiaceae considered as one of the most important fruits of the tropical and subtropical countries of the world. It grows under a wide range of climatic and soil conditions. In Egypt, mango ranks the second after citrus, whereas its total area of fruitful orchards reached approximately 285973 fed. producing about 1091535 tons annually (M.A.L.R., 2020). However, lower yield with poor quality is one of the main problems facing mango growers. Many trials were done to raise the productivity and fruit quality of much fruit species by the wasteful use of chemical fertilizers especially mineral ones. For avoiding the excessive use of the mineral chemical fertilizers and their harmful effect particularly on human health consequently, using of some natural biostimulants such as plant extracts that characterized by their richer contents with nutrient elements, growth regulators, antioxidants and vitamins were investigated for being applied as an effective nutritive 
application that could be replace partially or completely the aforesaid harmful ones (Spinelli et al., 2009; Ahmed et al., 2013 and Anwar et al., 2013).

Recent studies supported the beneficial effects of using crop seed sprouts to promote growth, tree nutrition status, yield and fruit quality in fruit crops and at the same time protecting our environment from pollution (Cazuola et al., 2004; Cairney, 2005; Biommerson, 2007 and Dhekney, 2016; Oraby, 2018 and Ali et al., 2018).

Previous studies showed using all nutrients via all forms were responsible for improving growth, yield and fruit quality of mangoes (Kassim and Marzouk, 2004; EbeidSanaa, 2007; Ibrahiem et al., 2007; El-Sayed-Esraa, 2007; El-SayedEsraa, 2010; Refaai, 2014; ElKhawaga and Mansour, 2014; Ahmed, 2015; Abdel-Rahman, 2015;
El-Sharony et al., 2015; Abdelaziz et al., 2015; Mohamed et al., 2015; Oraby, 2018 and Ali et al., 2018).

The aim of the work was examining the effect of foliar application of faba bean and chickpeas seed sprout on growth and fruiting of Ewaise mango trees grown under Upper Egypt conditions.

\section{Materials and Methods}

This investigation was carried out during 2019 and 2020 seasons on Ewaise mango cv. trees onto seeding rootstocks, grown in silty clay soil in a private orchard situated at El-Biora region, Kom Ombo district, Aswan governorate Egypt. The selected trees were 18- years old, healthy, nearly uniform in vigour, planted at $7 \times 7$ meters apart and received the same cultural practices. Surface irrigation system was used. Soil analysis was done according to the procedures outlined by Wilde et al. (1985) and the data are shown in Table (1).

Table 1. Analysis of the tested soil and chemical composition of fenugreek sprout.

\begin{tabular}{|c|c|c|c|}
\hline $\begin{array}{c}\text { Constituents } \\
\end{array}$ & Values & Constituents & Values (mg/100g F.W.) \\
\hline \multicolumn{2}{|l|}{ Particle size distribution } & Faba bean & \\
\hline Sand \% & 10.60 & Folate & 0.64 \\
\hline Silt \% & 32.80 & Niacin & 31.40 \\
\hline Clay $\%$ & 56.60 & Protein & 4500.00 \\
\hline Texture grade & Silty clay & Riboflavin & 2.78 \\
\hline $\mathrm{pH}(1: 2.5$ extract $)$ & 7.88 & Thiamin & 3.98 \\
\hline E.C. $(1: 2.5$ extract $)$ & 0.91 & Vitamin $B_{6}$ & 0.91 \\
\hline Organic matter \% & 2.15 & Vitamin C & 416.00 \\
\hline $\mathrm{CaCO}_{3} \%$ & 1.88 & Chickpea & \\
\hline Total N \% & 0.12 & Thiamin & 0.486 \\
\hline $\mathrm{P}($ Olsen, ppm) & 22.00 & Riboflavin & 0.106 \\
\hline $\mathrm{K}$ (ammonium acetate, $\mathrm{ppm}$ ) & 420.00 & Folate & 0.44 \\
\hline $\mathrm{Mg}(\mathrm{ppm})$ & 79.00 & Vitamin $\mathrm{B}_{6}$ & 0.492 \\
\hline $\mathrm{S}(\mathrm{ppm})$ & 6.95 & Vitamin A (International units) & 41.00 \\
\hline EDTA extractable & & Potassium & 846.00 \\
\hline $\mathrm{Zn}(\mathrm{ppm})$ & 1.36 & Calcium & 45.00 \\
\hline $\mathrm{Fe}(\mathrm{ppm})$ & 11.11 & Sodium & 64.00 \\
\hline $\mathrm{Mn}(\mathrm{ppm})$ & 10.25 & Zinc & 2.81 \\
\hline $\mathrm{Cu}(\mathrm{ppm})$ & 1.63 & & \\
\hline
\end{tabular}


This study included the following seven treatments:

1- Control (water).

2- Spraying faba bean seed sprout extract at $0.25 \%$

3- Spraying faba bean seed sprout extract at $0.50 \%$.

4- Spraying faba bean seed sprout extract at $1.0 \%$.

5- Spraying chickpeas seed sprout extract at $0.25 \%$.

6- Spraying chickpeas seed sprout extract at $0.50 \%$.

7- Spraying chickpeas seed sprout extract at $1.0 \%$.

The experiment was set up as a complete randomized block design. Each treatment was replicated three times, one tree per each. Faba bean and chickpeas seed sprout extract was prepared by sowing the seeds in dark place, then sprouts were harvested after 3-4 days from seed sowing. Sprouts were homogenated with distilled water at 1: 10 using an electric blender for five minutes, then filterated and kept under $4^{\circ} \mathrm{C}$ in refrigerator till use.

Faba bean and chickpeas seeds sprout extract were sprayed three times at growth start (first week of March) after fruit set immediately (third week of April) one month later (third week of May) in both seasons. Analysis of this extract was carried out using the procedures methods that outlined by A.O.A.C. (2000) and data are shown in Table (1). Triton B as a wetting agent was added to all spraying solutions at $0.05 \%$, and spraying was done till runoff.

During both seasons, the following parameters were measured, spring shoot length $(\mathrm{cm})$, shoot thickness $(\mathrm{cm})$, leaves number/shoot, leaf area $\left(\mathrm{cm}^{2}\right)$ (Ahmed and Morsy, 1999), total chlorophylls and total carotenoids as $\mathrm{mg} / 100$ g $\quad$ F.W. (Hiscox and Isralstam, 1979); percentages of nutritional elements (Wilde et al., 1985), Fruit retention\%, number of fruit/tree, total yield/tree $(\mathrm{kg})$, fruit weight (g), pulp percentage; T.S.S.\%, total acidity, sugars contents $\%$, vitamin $\mathrm{C}$ content $\mathrm{mg} / 100 \mathrm{ml}$ juice and total fibres (A.O.A.C., 2000).

Statistical analysis was done using new L.S.D. test at $5 \%$ to differentiate among the seven treatment means (Gomez \& Gomez, 1984 and Mead et al., 1993).

\section{Results}

1- Vegetative growth and leaf chemical composition

Tables 2, 3 \& 4 showed the effect of plant seed sprout extracts spraying on the length and thickness of shoot, number of leaves/shoot and leaf area as well as total of chlorophylls and carotenoids and leaf $\mathrm{N}, \mathrm{P}, \mathrm{K}, \mathrm{Mg}, \mathrm{Ca}, \mathrm{Zn}, \mathrm{Fe}$ and $\mathrm{Mn}$ contents of Ewaise mango trees during 2019 and 2020 seasons. It is obvious that the results showed a similar trend during the two studied seasons. Such results indicate that the spraying faba bean and chickpeas seed sprout extract significantly increased such traits compared to water sprayed (control). The promotion on such growth traits was associated with increasing the extract used concentration from 0.25 to $1.0 \%$. Increasing concentrations of faba bean or chickpeas seed sprout extract from 0.50 to $1.0 \%$ failed significantly to stimulate these 
studied growth and leaf traits. Therefore, from economical point view, it is the best to use faba bean or chickpeas seed sprout extract at $0.50 \%$. Moreover, using chickpeas significantly stimulated the shoot growth and leaf traits area as well as total chlorophylls, carotenoids and nutrient contents of leaves more than using faba bean seed sprout. The maximum values of shoot growth, as well as leaf traits and leaf nutrient contents were recorded on the trees that were spraying chickpeas at $1 \%$. Thus the highest values were $(18.4$ $\mathrm{cm}, 0.85 \mathrm{~cm}, 19.2$ leaf, $84.9 \mathrm{~cm}^{2}, 8.94$ $\mathrm{mg} / \mathrm{g}, 2.33 \%, 1.85 \%, 0.211 \%, 1.23 \%$, $0.67,3.03,65.2 \mathrm{ppm}, 67.38 \mathrm{ppm}$ and $57.83 \mathrm{ppm}$ as an av. two studied seasons for shoot length, shoot thickness, leaf no/shoot, leaf area, total chlorophylls, carotenoids, N, P, $\mathrm{K}, \mathrm{Mg}, \mathrm{Ca}, \mathrm{Zn}, \mathrm{Fe}$ and $\mathrm{Mn}$ ppm, respectively. On other hand, the lowest values of the growth traits as well as leaf total chlorophylls, total carotenoids and leaf nutrient contents were recorded on the trees that were sprayed with water (check trees). The corresponding least values were $16.2 \mathrm{~cm}, 0.61 \mathrm{~cm}, 12.9$ leaf, $78.0 \mathrm{~cm}^{2}$, $5.58 \mathrm{mg} / \mathrm{g}, 1.27 \%, 1.62 \%, 0.118 \%$, $1.06 \%, 0.50 \%, 2.76 \%, 59.5 \mathrm{ppm}$, $60.06 \mathrm{ppm}$ and $50.48 \mathrm{ppm}$, respectively. Then, the attained increment were $13.58,39.34,48.84$, $8.85,60.22,83.46,14.20,78.81$, $16.04,34.0,9.78,9.58,12.19$ and $14.56 \%$, respectively. Therefore, spraying faba bean or chickpeas seed sprout extract significantly increased the total leaf surface area, nutritional status and vegetative growth of mango trees.

\section{2 - Yield components}

It is clear from Table 5 that the spraying of Ewaise mango trees with faba bean or chickpeas sprout extract significantly increased the mango yield components, i.e. fruit retention $\%$, number of fruits/tree and total yield/tree compared to water sprayed (check treatment). The promotion in the yield component was associated with increasing the applied level concentration of extract used. No significant difference found due to increase concentration of extracts used from 0.5 to $1 \%$, thus from economical view it must be use the sprout extract at 0.50 to get the high economical yield. Using chickpeas seed sprout extract showed more significant increase in the yield than using faba seed sprout extract. The heaviest total yield/tree was recorded on the trees that sprayed with $1 \%$ chickpeas extract. The obtained values of total yield/tree were (42.9, $47.1,50.9,51.6,49.2,52.9$ and 53.6 $\mathrm{kg} /$ tree) due to spray with water (control) $\left(\mathrm{T}_{1}\right)$, faba bean seed sprout at $0.25\left(\mathrm{~T}_{2}\right), 0.50\left(\mathrm{~T}_{3}\right) \& 1 \%\left(\mathrm{~T}_{4}\right)$, chickpeas seed sprout at $0.25\left(\mathrm{~T}_{5}\right)$, $0.50\left(\mathrm{~T}_{6}\right)$ and $1 \%\left(\mathrm{~T}_{7}\right)$, respectively as an average of two studied seasons. The obtained increment of yield/tree as averages of two seasons was 9.79, $18.65,20.28,14.69,23.31 \& 24.94$ as a result of using $\mathrm{T}_{2}, \mathrm{~T}_{3}, \mathrm{~T}_{4}, \mathrm{~T}_{5}, \mathrm{~T}_{6}$ and $\mathrm{T}_{7}$, respectively, compared to $\mathrm{T}_{1}$ (check treatment). Therefore, it is clear that spraying with plant seed sprout extract on mango trees has beneficial effects. 
Table 2. Effect of spraying faba bean and chickpeas seed sprouts on some shoot and leaf traits of Ewaise mango trees during 2019 and 2020 seasons.

\begin{tabular}{|l|c|c|c|c|c|c|c|c|c|c|c|c|}
\hline \multirow{2}{*}{ Treat. } & \multicolumn{3}{|c|}{ Shoot length (cm) } & \multicolumn{3}{c|}{ Shoot thickness $(\mathbf{c m})$} & \multicolumn{3}{c|}{ No. of leaves/shoot } & \multicolumn{3}{c|}{ Leaf area $\left.\mathbf{c m}^{2}\right)$} \\
\cline { 2 - 14 } & $\mathbf{2 0 1 9}$ & $\mathbf{2 0 2 0}$ & Mean & $\mathbf{2 0 1 9}$ & $\mathbf{2 0 2 0}$ & Mean & $\mathbf{2 0 1 9}$ & $\mathbf{2 0 2 0}$ & Mean & $\mathbf{2 0 1 9}$ & $\mathbf{2 0 2 0}$ & Mean \\
\hline Control (water) & $15.3 \mathrm{~B}$ & $17.1 \mathrm{C}$ & 16.2 & $0.58 \mathrm{C}$ & $0.63 \mathrm{D}$ & 0.61 & $11.9 \mathrm{E}$ & $13.8 \mathrm{E}$ & 12.9 & $76.1 \mathrm{C}$ & $79.8 \mathrm{~B}$ & 78.0 \\
\hline Faba bean 0.25\% & $15.8 \mathrm{~B}$ & $18.0 \mathrm{BC}$ & 16.9 & $0.63 \mathrm{C}$ & $0.68 \mathrm{D}$ & 0.66 & $13.4 \mathrm{D}$ & $15.0 \mathrm{D}$ & 14.2 & $78.6 \mathrm{BC}$ & $81.9 \mathrm{~B}$ & 80.3 \\
\hline Faba bean 0.50\% & $16.2 \mathrm{AB}$ & $18.9 \mathrm{AB}$ & 17.6 & $0.72 \mathrm{~B}$ & $0.74 \mathrm{C}$ & 0.73 & $14.4 \mathrm{CD}$ & $16.1 \mathrm{C}$ & 15.3 & $81.5 \mathrm{~A}$ & $84.2 \mathrm{AB}$ & 82.9 \\
\hline Faba bean 1.0\% & $16.4 \mathrm{AB}$ & $19.7 \mathrm{~A}$ & 18.1 & $0.75 \mathrm{~B}$ & $0.78 \mathrm{BC}$ & 0.77 & $15.2 \mathrm{C}$ & $17.0 \mathrm{C}$ & 16.1 & $82.3 \mathrm{AB}$ & $84.9 \mathrm{~A}$ & 83.6 \\
\hline Chickpeas 0.25\% & $16.2 \mathrm{AB}$ & $18.7 \mathrm{~B}$ & 17.5 & $0.77 \mathrm{~B}$ & $0.81 \mathrm{~B}$ & 0.79 & $16.4 \mathrm{~B}$ & $18.3 \mathrm{~B}$ & 17.4 & $80.8 \mathrm{~B}$ & $83.2 \mathrm{~B}$ & 82.0 \\
\hline Chickpeas 0.50\% & $16.9 \mathrm{~A}$ & $19.6 \mathrm{~A}$ & 18.3 & $0.80 \mathrm{AB}$ & $0.83 \mathrm{AB}$ & 0.82 & $17.6 \mathrm{~A}$ & $19.8 \mathrm{~A}$ & 18.7 & $82.7 \mathrm{AB}$ & $85.9 \mathrm{~A}$ & 84.3 \\
\hline Chickpeas 1.0\% & $17.0 \mathrm{~A}$ & $19.8 \mathrm{~A}$ & 18.4 & $0.83 \mathrm{~A}$ & $0.86 \mathrm{~A}$ & 0.85 & $18.3 \mathrm{~A}$ & $20.1 \mathrm{~A}$ & 19.2 & $83.7 \mathrm{~A}$ & $86.0 \mathrm{~A}$ & 84.9 \\
\hline New LSD 5\% & $\mathbf{0 . 8 2}$ & $\mathbf{0 . 9 8}$ & & $\mathbf{0 . 0 5}$ & $\mathbf{0 . 0 5}$ & & $\mathbf{1 . 0 2}$ & $\mathbf{1 . 0 6}$ & & $\mathbf{2 . 8 1}$ & $\mathbf{2 . 6 8}$ & \\
\hline
\end{tabular}

Table 3. Effect of spraying faba bean and chickpeas sprouts on some leaf chemical composition of Ewaise mango trees during 2019 and 2020 seasons.

\begin{tabular}{|c|c|c|c|c|c|c|c|c|c|c|c|c|c|c|c|}
\hline \multirow{2}{*}{ Treat. } & \multicolumn{3}{|c|}{ Total chlorophylls } & \multicolumn{3}{|c|}{ Total carotenoids } & \multicolumn{3}{|c|}{ N\% } & \multicolumn{3}{|c|}{ P\% } & \multicolumn{3}{|c|}{ K\% } \\
\hline & 2019 & \begin{tabular}{|l|}
2020 \\
\end{tabular} & Mean & 2019 & 2020 & Mean & 2019 & 2020 & Mean & 2019 & 2020 & Mean & 2019 & 2020 & Mean \\
\hline $\begin{array}{l}\text { Control } \\
\text { (water) }\end{array}$ & $5.09 \mathrm{D}$ & $6.06 \mathrm{D}$ & 5.58 & $1.10 \mathrm{E}$ & $1.43 \mathrm{D}$ & 1.27 & $1.60 \mathrm{D}$ & $1.64 \mathrm{D}$ & 1.62 & $0.108 \mathrm{E}$ & $0.127 \mathrm{E}$ & 0.118 & $1.05 \mathrm{D}$ & $1.06 \mathrm{D}$ & 1.06 \\
\hline $\begin{array}{l}\text { Faba bean } \\
0.25 \%\end{array}$ & $6.03 \mathrm{C}$ & $6.82 \mathrm{C}$ & 6.43 & $1.35 \mathrm{D}$ & $1.58 \mathrm{D}$ & 1.47 & $1.66 \mathrm{C}$ & $1.69 \mathrm{C}$ & 1.68 & $0.141 \mathrm{D}$ & $0.158 \mathrm{D}$ & 0.150 & $1.10 \mathrm{C}$ & $1.14 \mathrm{C}$ & 1.12 \\
\hline $\begin{array}{ll}\text { Faba } & \text { bean } \\
\mathbf{0 . 5 0 \%} & \end{array}$ & $6.81 \mathrm{~B}$ & 7.74B & 7.28 & $1.68 \mathrm{C}$ & $1.95 \mathrm{C}$ & 1.82 & $1.73 \mathrm{~B}$ & $1.75 \mathrm{~B}$ & 1.74 & $0.188 \mathrm{~B}$ & $0.204 \mathrm{~B}$ & 0.196 & $1.16 \mathrm{~B}$ & $1.20 \mathrm{~B}$ & 1.18 \\
\hline $\begin{array}{l}\text { Faba bean } \\
1.0 \%\end{array}$ & $7.18 \mathrm{~B}$ & 8.03B & 7.61 & $1.80 \mathrm{C}$ & $2.05 \mathrm{BC}$ & 1.93 & $1.76 \mathrm{~B}$ & $1.79 \mathrm{~B}$ & 1.78 & $0.193 B$ & $0.210 \mathrm{~A}$ & 0.202 & $1.18 \mathrm{~A}$ & $1.22 \mathrm{~B}$ & 1.20 \\
\hline $\begin{array}{l}\text { Chickpeas } \\
0.25 \%\end{array}$ & $6.95 \mathrm{~B}$ & $7.76 \mathrm{~B}$ & 7.36 & $1.92 \mathrm{BC}$ & 2.19B & 2.06 & 1.74B & $1.76 \mathrm{~B}$ & 1.75 & $0.174 \mathrm{C}$ & $0.189 \mathrm{C}$ & 0.182 & $1.15 \mathrm{~B}$ & 1.1B8 & 1.17 \\
\hline $\begin{array}{l}\text { Chickpeas } \\
0.50 \%\end{array}$ & $8.26 \mathrm{~A}$ & $8.93 \mathrm{~A}$ & 8.60 & $2.13 \mathrm{~A}$ & $2.38 \mathrm{~A}$ & 2.26 & $1.81 \mathrm{~A}$ & $1.84 \mathrm{~A}$ & 1.83 & $0.199 \mathrm{AB}$ & $0.211 \mathrm{~A}$ & 0.205 & $1.20 \mathrm{~A}$ & $1.25 \mathrm{AB}$ & 1.23 \\
\hline $\begin{array}{l}\text { Chickpeas } \\
1.0 \%\end{array}$ & $8.53 \mathrm{~A}$ & $9.33 \mathrm{~A}$ & 8.94 & $2.18 \mathrm{~A}$ & $2.48 \mathrm{~A}$ & 2.33 & $1.85 \mathrm{~A}$ & $1.85 \mathrm{~A}$ & 1.85 & $0.206 \mathrm{~A}$ & $0.216 \mathrm{~A}$ & 0.211 & $1.19 \mathrm{~A}$ & $1.26 \mathrm{~A}$ & 1.23 \\
\hline New LSD 5\% & 0.51 & 0.49 & & 0.12 & 0.14 & & 0.05 & 0.05 & & 0.011 & 0.012 & & 0.04 & 0.04 & \\
\hline
\end{tabular}

Table 4. Effect of spraying faba bean and chickpeas sprouts on some leaf nutrients of Ewaise mango trees during 2019 and 2020 seasons.

\begin{tabular}{|c|c|c|c|c|c|c|c|c|c|c|c|c|c|c|c|}
\hline \multirow{2}{*}{ Treat. } & \multicolumn{3}{|c|}{ Mg \% } & \multicolumn{3}{|c|}{$\mathrm{Ca} \%$} & \multicolumn{3}{|c|}{ Zn ppm } & \multicolumn{3}{|c|}{ Fe ppm } & \multicolumn{3}{|c|}{ Mn ppm } \\
\hline & 2019 & 2020 & Mean & 2019 & \begin{tabular}{|l|}
2020 \\
\end{tabular} & Mean & 2019 & 2020 & Mean & 2019 & 2020 & Mean & 2019 & 2020 & Mean \\
\hline $\begin{array}{l}\text { Control } \\
\text { (water) }\end{array}$ & $0.50 \mathrm{D}$ & $0.50 \mathrm{D}$ & 0.50 & $2.71 \mathrm{C}$ & $2.80 \mathrm{C}$ & 2.76 & $60.8 \mathrm{D}$ & $58.1 \mathrm{C}$ & 59.5 & $58.30 \mathrm{D}$ & $61.82 \mathrm{C}$ & 60.06 & 49.33D & $51.63 \mathrm{C}$ & 50.48 \\
\hline $\begin{array}{l}\text { Faba bean } \\
0.25 \%\end{array}$ & $0.55 \mathrm{C}$ & $0.57 \mathrm{C}$ & 0.56 & $2.85 \mathrm{~B}$ & $2.90 \mathrm{~B}$ & 2.88 & $63.3 \mathrm{C}$ & $61.4 \mathrm{~B}$ & 62.4 & $60.81 \mathrm{C}$ & $63.38 \mathrm{C}$ & 62.10 & $52.54 \mathrm{C}$ & $54.28 \mathrm{~B}$ & 53.41 \\
\hline $\begin{array}{l}\text { Faba bean } \\
\mathbf{0 . 5 0 \%}\end{array}$ & $0.60 \mathrm{~B}$ & $0.62 \mathrm{~B}$ & 0.61 & $2.9 \mathrm{~B}$ & $2.99 \mathrm{AB}$ & 2.96 & $64.7 \mathrm{BC}$ & $63.6 \mathrm{~A}$ & 64.2 & 63.34B & $66.21 \mathrm{~B}$ & 64.78 & $54.63 \mathrm{BC}$ & $56.79 \mathrm{~A}$ & 55.71 \\
\hline $\begin{array}{ll}\text { Faba } & \text { bean } \\
1.0 \% & \end{array}$ & $0.61 \mathrm{~B}$ & $0.63 \mathrm{~B}$ & 0.62 & $2.95 \mathrm{AB}$ & $3.00 \mathrm{AB}$ & 2.98 & $65.5 \mathrm{~B}$ & $63.8 \mathrm{~A}$ & 64.7 & $63.92 \mathrm{~B}$ & $66.64 \mathrm{~A}$ & 65.28 & $55.58 \mathrm{~B}$ & $57.26 \mathrm{~A}$ & 56.42 \\
\hline $\begin{array}{l}\text { Chickpeas } \\
0.25 \%\end{array}$ & $0.60 \mathrm{~B}$ & $0.62 \mathrm{~B}$ & 0.61 & $2.91 \mathrm{~B}$ & $2.92 \mathrm{~B}$ & 2.92 & $64.1 \mathrm{BC}$ & $60.9 \mathrm{~B}$ & 62.5 & $62.11 \mathrm{BC}$ & $64.67 \mathrm{BC}$ & 63.39 & $53.18 \mathrm{C}$ & $54.58 \mathrm{~B}$ & 53.88 \\
\hline $\begin{array}{l}\text { Chickpeas } \\
0.50 \%\end{array}$ & $0.65 \mathrm{~A}$ & $0.67 \mathrm{~A}$ & 0.66 & $2.99 \mathrm{AB}$ & $3.06 \mathrm{~A}$ & 3.03 & $66.7 \mathrm{AB}$ & $63.6 \mathrm{~A}$ & 65.2 & $65.68 \mathrm{AB}$ & $68.65 \mathrm{~A}$ & 67.17 & $56.82 \mathrm{AB}$ & $57.60 \mathrm{~A}$ & 57.21 \\
\hline $\begin{array}{l}\text { Chickpeas } \\
1.0 \%\end{array}$ & $0.66 \mathrm{~A}$ & $0.68 \mathrm{~A}$ & 0.67 & $3.03 \mathrm{~A}$ & $3.03 \mathrm{~A}$ & 3.03 & $68.1 \mathrm{~A}$ & $62.2 \mathrm{~A}$ & 65.2 & $66.53 \mathrm{~A}$ & $68.23 \mathrm{~A}$ & 67.38 & $57.80 \mathrm{~A}$ & $57.85 \mathrm{~A}$ & 57.83 \\
\hline $\begin{array}{ll}\text { New } & \text { LSD } \\
\mathbf{5 \%} & \end{array}$ & 0.03 & 0.03 & & 0.10 & 0.09 & & 1.93 & 2.11 & & 2.23 & 2.15 & & 1.65 & 1.52 & \\
\hline
\end{tabular}


Table 5. Effect of spraying faba bean and chickpeas sprouts on yield components and fruit weight of Ewaise mango trees during 2019 and 2020 seasons.

\begin{tabular}{|l|c|c|c|c|c|c|c|c|c|c|c|c|}
\hline \multicolumn{1}{|c|}{ Treat. } & \multicolumn{2}{|c|}{ Fruit retention } & \multicolumn{3}{c|}{ No. of fruits/tree } & \multicolumn{3}{c|}{ Total yield/tree (kg) } & \multicolumn{3}{c|}{ Fruit weight } \\
\hline & $\mathbf{2 0 1 9}$ & $\mathbf{2 0 2 0}$ & Mean & $\mathbf{2 0 1 9}$ & $\mathbf{2 0 2 0}$ & Mean & $\mathbf{2 0 1 9}$ & $\mathbf{2 0 2 0}$ & Mean & $\mathbf{2 0 1 9}$ & $\mathbf{2 0 2 0}$ & Mean \\
\hline Control (water) & $0.61 \mathrm{~B}$ & $0.70 \mathrm{~B}$ & 0.66 & $223.8 \mathrm{C}$ & $235.9 \mathrm{D}$ & 229.9 & $41.5 \mathrm{E}$ & $44.2 \mathrm{E}$ & 42.9 & $185.8 \mathrm{C}$ & $187.4 \mathrm{C}$ & 186.6 \\
\hline Faba bean 0.25\% & $0.68 \mathrm{~A}$ & $0.79 \mathrm{~A}$ & 0.74 & $234.9 \mathrm{~B}$ & $249.8 \mathrm{C}$ & 242.4 & $45.1 \mathrm{D}$ & $49.1 \mathrm{D}$ & 47.1 & $192.3 \mathrm{~B}$ & $196.9 \mathrm{~B}$ & 194.6 \\
\hline Faba bean 0.50\% & $0.69 \mathrm{~A}$ & $0.77 \mathrm{~A}$ & 0.73 & $247.1 \mathrm{AB}$ & $262.3 \mathrm{~B}$ & 254.7 & $48.6 \mathrm{~B}$ & $53.1 \mathrm{~B}$ & 50.9 & $197.8 \mathrm{~A}$ & $203.1 \mathrm{AB}$ & 200.5 \\
\hline Faba bean 1.0\% & $0.69 \mathrm{~A}$ & $0.77 \mathrm{~A}$ & 0.73 & $248.5 \mathrm{AB}$ & $264.1 \mathrm{~A}$ & 256.3 & $49.3 \mathrm{~B}$ & $53.9 \mathrm{~B}$ & 51.6 & $199.6 \mathrm{~A}$ & $204.8 \mathrm{~A}$ & 202.2 \\
\hline Chickpeas 0.25\% & $0.68 \mathrm{~A}$ & $0.76 \mathrm{~A}$ & 0.72 & $241.4 \mathrm{~B}$ & $256.5 \mathrm{BC}$ & 249.0 & $47.1 \mathrm{C}$ & $51.2 \mathrm{C}$ & 49.2 & $195.3 \mathrm{~A}$ & $200.9 \mathrm{~B}$ & 198.1 \\
\hline Chickpeas 0.50\% & $0.70 \mathrm{~A}$ & $0.78 \mathrm{~A}$ & 0.74 & $252.6 \mathrm{~A}$ & $271.8 \mathrm{AB}$ & 262.2 & $49.8 \mathrm{AB}$ & $55.9 \mathrm{~A}$ & 52.9 & $198.9 \mathrm{~A}$ & $206.5 \mathrm{AB}$ & 202.7 \\
\hline Chickpeas 1.0\% & $0.69 \mathrm{~A}$ & $0.77 \mathrm{~A}$ & 0.73 & $253.2 \mathrm{~A}$ & $273.1 \mathrm{~A}$ & 263.2 & $50.7 \mathrm{~A}$ & $56.5 \mathrm{~A}$ & 53.6 & $200.8 \mathrm{~A}$ & $208.8 \mathrm{~A}$ & 204.8 \\
\hline New LSD 5\% & $\mathbf{0 . 0 5}$ & $\mathbf{0 . 0 6}$ & & $\mathbf{9 . 3 9}$ & $\mathbf{1 0 . 3 1}$ & & $\mathbf{1 . 1 8}$ & $\mathbf{1 . 9 0}$ & & $\mathbf{6 . 4 2}$ & $\mathbf{7 . 2 8}$ & \\
\hline
\end{tabular}

\section{3- Fruit Quality}

It is evident from Tables $6,7 \&$ 8 that treating faba bean and chickpeas seed sprout extract at 0.25 to $1 \%$ significantly improved the fruit quality in terms of increasing the fruit weight, pulp \%, T.S.S. $\%$ and sugar contents as well as, vitamin $\mathrm{C}$ content and decreasing the total acidity and total fiber compared to untreated ones (control). The spraying of mango trees with 0.25 to $1 \%$ chickpeas seed sprout extract significantly improved the fruit quality more than using 0.25 to $1 \%$ faba bean seed sprout extract. The highest values of mango fruit traits were recorded on the trees that sprayed with $1.0 \%$ chickpeas seed sprout extract and compared to other treatments and untreated ones (control). On other hand, the lowest values of fruit quality were found on the trees that sprayed with water (control). No significant differences were found due to increase the concentration of any extract used from 0.50 to $1 \%$.

The highest fruit weight $(204.8$ g), pulp \% (72.15\%), TSS (16.60\%), total sugar $(10.39 \%)$, V.C (48.0 $\mathrm{mg} / \mathrm{g})$ and total acidity $(0.294 \%)$ due to spray chickpea at $1 \%$ chickpeas seed sprout extract. On other hand, the lowest corresponding values were (186.6 g, 64.04\%, 15.33\%, 9.50\%, $41.0 \mathrm{mg} / \mathrm{g}$ and $0.352 \%$ ), respectively, due to water sprayed (control).

Hence, the increment percentages of these traits were 9.75 , $12.66,8.25,9.37$ and $17.07 \%$ due to using $1 \%$ chickpeas, respectively. In addition, the respective decrement of total acidity was attained 16.48\%.compared to (check treatment).

Hence, the cost wise evaluation of the application of faba bean or chickpeas at $0.5 \%$ three times. Such applications is very important for the production of mango fruits, since improving of the fruit quality induces an increase in the packable yield.

\section{Discussion}

Crop the higher content of all seed sprouts from nutrients amino acids, vitamins, antioxidant and plant pigments surely reflected an enhancing growth tree, nutritional status, yield and fruit quality (Cazuola, 2004 and Cairney, 2005).

In addition, germination of seeds lead to enhance the availability of most organic and mineral nutrients (Biommerson, 2007; Anwar et al., 
2013 and Dhekney, 2016) the promotion on growth and tree nutrition of status surely reflected on enhancing yield and fruit quality. Our results indicated that treating the Ewaise mango trees with faba bean and chickpeas seed sprout extract at $0.5 \%$ three time improved the growth and fruiting of trees which increased the leaf area about $7.2 \%$ total chlorophyll 42.3\%, N 10.2\%, K 13\%, yield $20 \%$, fruit weight $8.3 \%$, total sugars $8 \%$ and vitamin $\mathrm{C}$ about $16.0 \%$. These results are in harmony with those obtained by Ebeid-Sanaa, 2007; Ibrahim et al., 2007; El-SayedEsraa, 2010; Refaai, 2014; ElKhawaga and Mansour, 2014; Ahmed, 2015; El-Sharony et al., 2015; Abdelaziz et al., 2015; Mohamed et al., 2015; Oraby, 2018 and Ali et al., 2018.

Table 6. Effect of spraying faba bean and chickpeas sprouts on some fruit quality of Ewaise mango trees during 2019 and 2020 seasons.

\begin{tabular}{|l|c|c|c|c|c|c|c|c|c|c|c|c|}
\hline \multirow{2}{*}{ Treat. } & \multicolumn{3}{|c|}{ Fruit pulp \% } & \multicolumn{4}{c|}{ TSS $\wedge$} & \multicolumn{3}{c|}{ Total sugar \% } & \multicolumn{3}{c|}{ Reducing \% } \\
\cline { 2 - 13 } & $\mathbf{2 0 1 9}$ & $\mathbf{2 0 2 0}$ & Mean & $\mathbf{2 0 1 9}$ & $\mathbf{2 0 2 0}$ & Mean & $\mathbf{2 0 1 9}$ & $\mathbf{2 0 2 0}$ & Mean & $\mathbf{2 0 1 9}$ & $\mathbf{2 0 2 0}$ & Mean \\
\hline Control (water) & $64.45 \mathrm{C}$ & 63.63 & 64.04 & $15.12 \mathrm{C}$ & $15.53 \mathrm{C}$ & 15.33 & $9.35 \mathrm{C}$ & $9.65 \mathrm{D}$ & 9.50 & $2.72 \mathrm{C}$ & $2.78 \mathrm{C}$ & 2.75 \\
\hline Faba bean 0.25\% & $65.78 \mathrm{BC}$ & 66.36 & 66.07 & $15.53 \mathrm{C}$ & $15.76 \mathrm{C}$ & 15.65 & $9.68 \mathrm{~B}$ & $9.98 \mathrm{C}$ & 9.83 & $2.83 \mathrm{~B}$ & $2.90 \mathrm{~B}$ & 2.87 \\
\hline Faba bean 0.50\% & $68.93 \mathrm{AB}$ & 69.50 & 69.22 & $16.10 \mathrm{~B}$ & $16.38 \mathrm{AB}$ & 16.24 & $9.98 \mathrm{AB}$ & $10.32 \mathrm{~B}$ & 10.15 & $2.90 \mathrm{~A}$ & $2.99 \mathrm{AB}$ & 2.95 \\
\hline Faba bean 1.0\% & $69.28 \mathrm{AB}$ & 69.81 & 69.55 & $16.28 \mathrm{AB}$ & $16.35 \mathrm{~B}$ & 16.33 & $10.05 \mathrm{~A}$ & $10.40 \mathrm{AB}$ & 10.23 & $2.91 \mathrm{AB}$ & $2.98 \mathrm{AB}$ & 2.95 \\
\hline Chickpeas 0.25\% & $68.60 \mathrm{~B}$ & 68.48 & 68.24 & $15.95 \mathrm{~B}$ & $16.11 \mathrm{~B}$ & 16.03 & $9.73 \mathrm{~B}$ & $10.05 \mathrm{BC}$ & 9.89 & $2.83 \mathrm{~B}$ & $2.89 \mathrm{~B}$ & 2.86 \\
\hline Chickpeas 0.50\% & $71.83 \mathrm{~A}$ & 72.15 & 71.99 & $16.38 \mathrm{AB}$ & $16.62 \mathrm{AB}$ & 16.50 & $10.18 \mathrm{~A}$ & $10.55 \mathrm{AB}$ & 10.37 & $2.96 \mathrm{~A}$ & $3.05 \mathrm{~A}$ & 3.01 \\
\hline Chickpeas 1.0\% & $72.11 \mathrm{~A}$ & 72.18 & 72.15 & $16.50 \mathrm{~A}$ & $16.70 \mathrm{~A}$ & 16.60 & $10.15 \mathrm{~A}$ & $10.62 \mathrm{~A}$ & 10.39 & $2.96 \mathrm{~A}$ & $3.08 \mathrm{~A}$ & 3.02 \\
\hline New LSD 5\% & $\mathbf{3 . 1 8}$ & $\mathbf{3 . 0 6}$ & & $\mathbf{0 . 3 1}$ & $\mathbf{0 . 3 2}$ & & $\mathbf{0 . 2 6}$ & $\mathbf{0 . 2 8}$ & & $\mathbf{0 . 1 0}$ & $\mathbf{0 . 1 1}$ & \\
\hline
\end{tabular}

Table 7. Effect of spraying faba bean and chickpeas sprouts on some fruit quality of Ewaise mango trees during 2019 and 2020 seasons.

\begin{tabular}{|l|c|c|c|c|c|c|c|c|c|c|c|c|}
\hline \multirow{2}{*}{ Treat. } & \multicolumn{2}{|c|}{ Non-reducing \% } & \multicolumn{2}{c|}{ Tota acidity \% } & \multicolumn{2}{c|}{ V.C. mg/100 ml } & \multicolumn{3}{c|}{ Total fiber } \\
\cline { 2 - 15 } & $\mathbf{2 0 1 9}$ & $\mathbf{2 0 2 0}$ & Mean & $\mathbf{2 0 1 9}$ & $\mathbf{2 0 2 0}$ & Mean & $\mathbf{2 0 1 9}$ & $\mathbf{2 0 2 0}$ & Mean & $\mathbf{2 0 1 9}$ & $\mathbf{2 0 2 0}$ & Mean \\
\hline Control (water) & $6.63 \mathrm{D}$ & $6.87 \mathrm{D}$ & 6.75 & $0.345 \mathrm{~A}$ & $0.358 \mathrm{~A}$ & 0.352 & $41.2 \mathrm{C}$ & $40.8 \mathrm{C}$ & 41.0 & $1.11 \mathrm{~A}$ & $1.16 \mathrm{~A}$ & 1.14 \\
\hline Faba bean 0.25\% & $6.83 \mathrm{C}$ & $7.08 \mathrm{C}$ & 6.96 & $0.326 \mathrm{~B}$ & $0.318 \mathrm{~B}$ & 0.322 & $43.4 \mathrm{~B}$ & $45.3 \mathrm{~B}$ & 44.4 & $1.03 \mathrm{~B}$ & $0.99 \mathrm{~B}$ & 1.01 \\
\hline Faba bean 0.50\% & $7.08 \mathrm{~A}$ & $7.33 \mathrm{~B}$ & 7.20 & $0.308 \mathrm{C}$ & $0.299 \mathrm{C}$ & 0.304 & $46.1 \mathrm{~A}$ & $48.9 \mathrm{~A}$ & 47.5 & $0.96 \mathrm{C}$ & $0.92 \mathrm{C}$ & 0.94 \\
\hline Faba bean 1.0\% & $7.14 \mathrm{~A}$ & $7.42 \mathrm{AB}$ & 7.28 & $0.303 \mathrm{C}$ & $0.292 \mathrm{C}$ & 0.298 & $46.8 \mathrm{~A}$ & $48.5 \mathrm{~A}$ & 47.7 & $0.95 \mathrm{C}$ & $0.91 \mathrm{C}$ & 0.93 \\
\hline Chickpeas 0.25\% & $6.90 \mathrm{~B}$ & $7.16 \mathrm{BC}$ & 7.03 & $0.318 \mathrm{~B}$ & $0.310 \mathrm{~B}$ & 0.314 & $44.3 \mathrm{~B}$ & $46.1 \mathrm{~B}$ & 45.2 & $0.98 \mathrm{BC}$ & $0.94 \mathrm{BC}$ & 0.96 \\
\hline Chickpeas 0.50\% & $7.22 \mathrm{~A}$ & $7.50 \mathrm{~A}$ & 7.36 & $0.301 \mathrm{C}$ & $0.289 \mathrm{C}$ & 0.295 & $46.6 \mathrm{~A}$ & $48.5 \mathrm{~A}$ & 47.6 & $0.91 \mathrm{C}$ & $0.87 \mathrm{C}$ & 0.89 \\
\hline Chickpeas 1.0\% & $7.19 \mathrm{~A}$ & $7.54 \mathrm{~A}$ & 7.37 & $0.298 \mathrm{C}$ & $0.290 \mathrm{C}$ & 0.294 & $47.1 \mathrm{~A}$ & $48.8 \mathrm{~A}$ & 48.0 & $0.92 \mathrm{C}$ & $0.86 \mathrm{C}$ & 0.89 \\
\hline New LSD 5\% & $\mathbf{0 . 1 8}$ & $\mathbf{0 . 1 7}$ & & $\mathbf{0 . 0 1 2}$ & $\mathbf{0 . 0 1 2}$ & & $\mathbf{1 . 2 8}$ & $\mathbf{1 . 3 5}$ & & $\mathbf{0 . 0 6}$ & $\mathbf{0 . 0 6}$ & \\
\hline
\end{tabular}

\section{Conclusion}

Through the results obtained, it was found that both the extracts gives a significant effect compared to the control and spraying trees sprout three times at growth started, just after fruit setting and one month later of faba bean or chickpeas at $0.5 \%$ was responsible for improving or promoting yield and fruit quality of Ewaise mango trees under Aswan Governorate and it's similar conditions.

\section{References}


A.O.A.C. (2000). Official Methods of Analysis $16^{\text {th }}$ Ed. A.O.A.C. Benjamin Franklin Station, Washington, D.C., .S.A. pp. 490510 .

Abdelaziz; F.H.; M.A. Mohamed and S.E.M. Abd El-Rady (2015). Relation of fruiting in Ewaise mango trees to foliar application of royal jelly, magnesium and boron. World Rural Observ., 7(2): 85-92.

Abdel-Rahman M.M.A. (2015). Yield and fruit quality of Washington Navel oranges as influenced by foliar application of fenugreek and rocket seed sprouts World Rural Observations, 7(2): 99-104.

Ahmed, F.F. and M.H. Morsy, M.H. (1999). A new method for measuring leaf area in different fruit species. Minia. J., Agric. Res. \& Dev. 19: 97-105.

Ahmed, F.F.; A.E.M. Mansour; M.A.A. Montasser; M.A. Merwad and E.A.M. Mostafa (2013). Response of Valencia orange trees to foliar application of roselle, turmeric and seaweed extracts. J of Applied Sci. Res., 9: 960-964.

Ahmed, M.Y.A. (2015). Effect of spraying fenugreek sprout and some nutrients on fruiting of Keitte Mango trees grown under Aswan region conditions. Alex. J. Agric. Res. Alex, J. Agric. Res. 50 (2): 33-40.

Ali, A.H.; M.M. Refaai and R. Abd ElBaset-Heba (2018). Improving productivity of Ewaise mango trees by using seed sprout extract of wheat beside carboxylic acid enriched with some macro and micro nutrients. Researcher 10 (12): 30-38.

Anwar, S.A.; A.K. Hifnawy; A.M. Kandel and M.F. Abdallah (2013). Nutritional and health related constitutions of fenugreek, sunflower and mustared sprouts as a functional food .Annals Agric. Sci., 50(1): 175-189.

Biommerson, A. (2007). Cruciferous sprout complex. Monograph, 227 Bellevue way NE, pp 83

Cairney, E. (2005). The sprouters handbook Argyll publishing Glendranel, Argyll PA 223 A 223 A E Scotland pp. 41-45.

Cazuola, I.; V. Marsili and G.L.K. Gainfrancshi (2004). Synthesis of antioxidants in wheat sprouts. J. Agric. Chen. 52: 5201-5206.

Dhekney, S.A. (2016). Encyclopedia of food and health. Academic press, Oxfor. pp. 261-265.

Ebeid-Sanaa, M. (2007). The promotive effect of seaweed extract and boron on growth and fruiting of Hindy Bisinnara mango trees. Minia J. of Agric. Rev \& Develop., 27 (3): 579-594.

El-Khawaga, A.S. and A.G.M. Mansour (2014). Promoting productivity of Washington navel orange trees by using some crop seed sprout Extracts, silicon and Glutathione. Middle East Journal of Applied Sciences, 4(3): 779-785.

El-Sayed-Esraa, M.H. (2007). Response of Ewaise mango trees to foliar application of boron. M.Sc. Thesis Fac. of Agric. Minia Univ. Egypt.

El-Sayed-Esraa, M.H. (2010). Behaviour of Ewaise mango trees to foliar application of some nutrients and seaweed extract. Ph.D. Thesis Fac. of Agric. Minia Univ. Egypt.

El-Sharony, T.F.; S.F. El-Gioushy and O.A. Amin (2015). Effect of foliar application with algae and plant extracts on growth, yield and fruit quality of fruitful mango trees $\mathrm{cv}$. Fagri Kalan. J. of Horticulture 2 (4): 1-6.

Gomez, K.A. and A.A. Gomez (1984). Statistical Procedures for Agricultural Research, 2nd Ed. Willy, New York. 
Hiscox, A. and B. Isralstam (1979). Method for the extraction of chlorophyll from leaf tissue without maceration. Can. J. Bot. 57:1332-1334.

Ibrahiem, H.I.M.; Y.M. Ahmed and F.F. Ahmed (2007). Relation of fruiting in Hindy Bisinnara mangoes to foliar nutrition with $\mathrm{Mg}, \mathrm{B}$ and $\mathrm{Zn}$ and some antioxidants African Crop Sci. Conf. Proc., 8: 411-415.

Kassim, H.A. and H.A. Marzouk (2004). Fruit retention, yield and postharvest fruit quality of mango in relation to time and number of potassium nitrite, zinc and boron foliar sprays. J. Alex. Res. (Fac. Agric. Saba Basha) 9 (2):299-311.

Mead, R.; R.N. Currow and A.M. Harted (1993). Statistical Methods in Agricultural Biology. $2^{\text {nd }}$ Ed. Chapman \& Hall, London.pp.50 70.

Mohamed, M.A.; M.A. El-Sayed and H.A.M. Abd El-Wahab (2015). Response of Succary mango trees to foliar application of silicon and boron. World Rural Observations, 7(2):93-98.
Oraby, A.F. (2018). Response of Ewaise mango trees grown under upper Egypt conditions to foliar application of some nutrients, plant extracts, Silicon and Selenium. Ph.D. Thesis Fac. of Agric. Minia Univ. Egypt.

Refaai, M.M. (2014). Impact of Spraying Extracts of Fenugreek and Rocket Seed Sprouts on Fruiting of Keitte Mango Trees. World Rural Observations, 6(4), 75-80.

Spinelli, F.; F. Giovanni; N. Massimo; S. Mattia and C. Guglielmo (2009). Perspectives on the use of a sea weed extract to moderate the negative effects of alternate bearing in apple trees. J Hort. Sci. Biotechn., 17: 131-137.

Wilde, S.A.; R.B. Corey; J.G. Layer and G.K. Voigt (1985). Soil and plant analysis for tree culture. Oxford and IBH publishing Co., New Delhi, India.

Yearly of statistics and Agricultural Economic Dept. Ministry of Agric. and Reclamation (M.A.L.R.), Egypt (2020). 


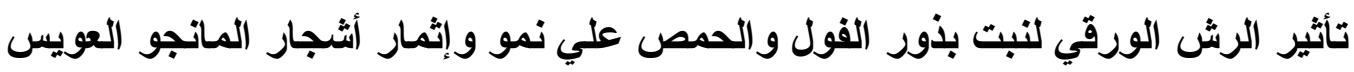

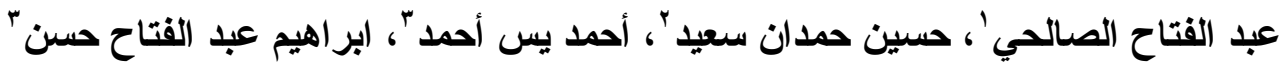

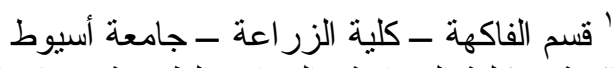

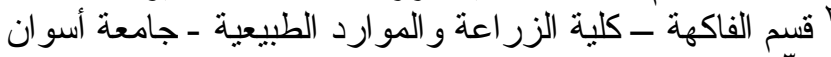

آمركز البحوث الزر اعية بمعهد البحوث البستانية بالجيزة البرة

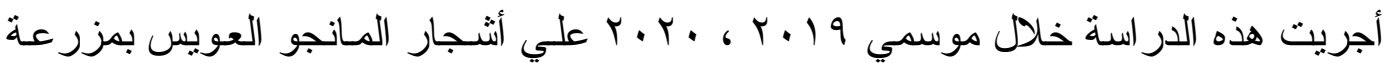

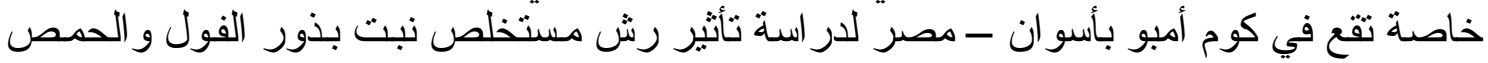

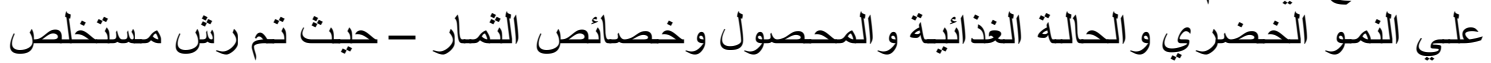

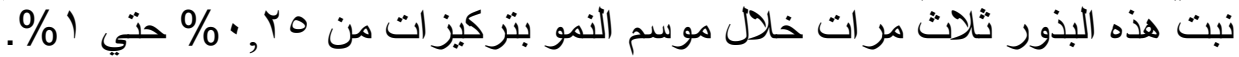

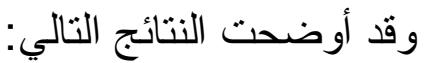

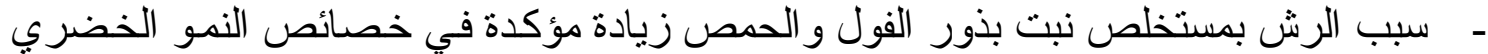

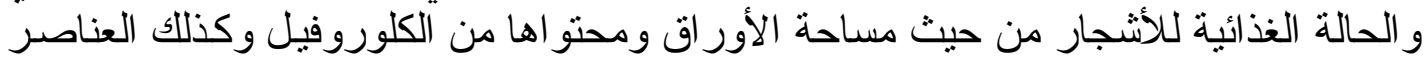

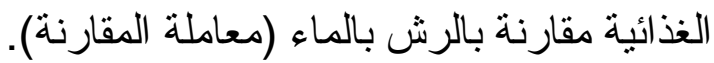

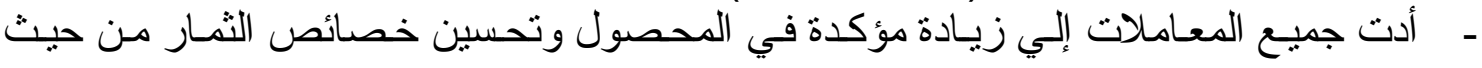

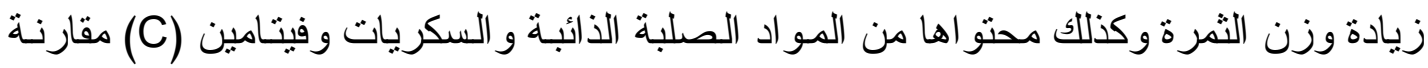

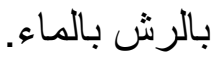

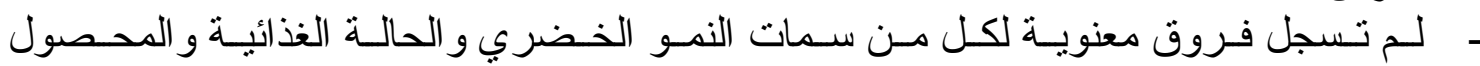

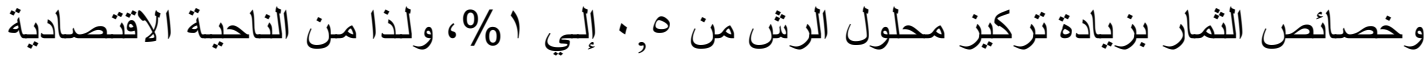

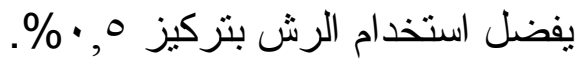

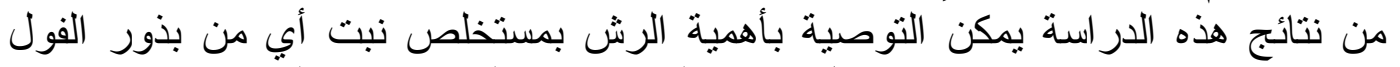

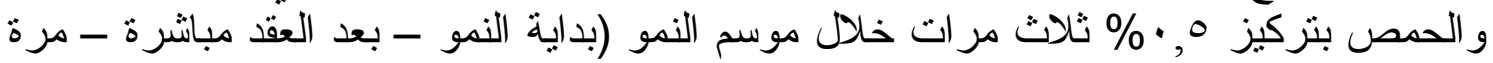

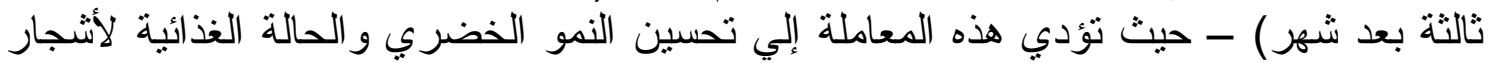

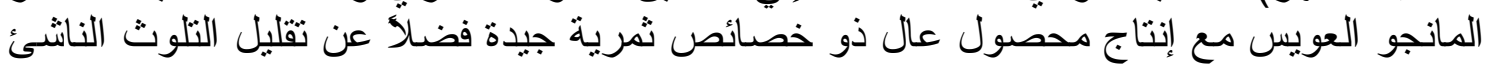
عن استخدام المو اد الكيماوية في البساتين حيث أنها مستخلصات طبية ديعية. 